\title{
Assessing 3D Tunnel Position in ACL Reconstruction Using A Novel Single Image 3D-2D Registration
}

\author{
X. Kang ${ }^{\mathrm{a}, \mathrm{b}}$, W.P. Yau ${ }^{\mathrm{a}}$, Y. Otake ${ }^{\mathrm{b}}$, P.Y.S. Cheung ${ }^{\mathrm{c}}$, Y. Hu ${ }^{\mathrm{a}}$, R.H. Taylor ${ }^{\mathrm{b}}$ \\ ${ }^{a}$ Dept. of Orthopaedics \& Traumatology, The University of Hong Kong, HK \\ ${ }^{\mathrm{b}}$ Dept. of Computer Science, Johns Hopkins University, Baltimore, MD, USA 21218 \\ ${ }^{c}$ Dept. of Electrical and Electronic Engineering, The University of Hong Kong, HK
}

\begin{abstract}
The routinely used procedure for evaluating tunnel positions following anterior cruciate ligament (ACL) reconstructions based on standard X-ray images is known to pose difficulties in terms of obtaining accurate measures, especially in providing three-dimensional tunnel positions. This is largely due to the variability in individual knee joint pose relative to X-ray plates. Accurate results were reported using postoperative CT. However, its extensive usage in clinical routine is hampered by its major requirement of having CT scans of individual patients, which is not available for most ACL reconstructions. These difficulties are addressed through the proposed method, which aligns a knee model to X-ray images using our novel single-image 3D-2D registration method and then estimates the 3D tunnel position. In the proposed method, the alignment is achieved by using a novel contour-based 3D-2D registration method wherein image contours are treated as a set of oriented points. However, instead of using some form of orientation weighting function and multiplying it with a distance function, we formulate the 3D-2D registration as a probability density estimation using a mixture of von Mises-Fisher-Gaussian (vMFG) distributions and solve it through an expectation maximization (EM) algorithm. Compared with the ground-truth established from postoperative CT, our registration method in an experiment using a plastic phantom showed accurate results with errors of $\left(-0.43^{\circ} \pm 1.19^{\circ}, 0.45^{\circ} \pm 2.17^{\circ}, 0.23^{\circ} \pm 1.05^{\circ}\right)$ and $(0.03 \pm 0.55$, $-0.03 \pm 0.54,-2.73 \pm 1.64) \mathrm{mm}$. As for the entry point of the ACL tunnel, one of the key measurements, it was obtained with high accuracy of $0.53 \pm 0.30 \mathrm{~mm}$ distance errors.
\end{abstract}

Keywords: 3D-2D registration, contour-based registration, expectation maximization, anterior cruciate ligament (ACL).

\section{INTRODUCTION}

The importance of tunnel placement in anterior cruciate ligament (ACL) reconstructions has received much attention recently since proper tunnel placement is essential with respect to both short-term and long-term outcomes. Validating the tunnel position is a prerequisite to the evaluation of clinical results following the ACL reconstruction. Thus, accurate assessment of the tunnel position is important for the evaluation. Most anatomical studies have involved cadaveric dissections that require destruction of the specimen as measurements are taken. This kind of studies cannot be used in clinical routine on live patients.

In clinical practice, tunnel position is routinely evaluated using postoperative anteroposterior (AP) and lateral (LAT) $\mathrm{X}$-ray. However, plain X-rays only provide $2 \mathrm{D}$ projections of the $3 \mathrm{D}$ anatomic bone geometry. Accurate measurement from them may be difficult to achieve reliably. Moreover, the entry points are hard to be accurately located manually due to its high dependency of the image on the rotation in the notch view. Assessment can also be performed intraoperatively using fluoroscopic images. But it has been shown ${ }^{1}$ that the intraoperative fluoroscopic measurements were not sufficient for the decision with respect to tunnel placement. These difficulties introduce errors in the direct correlation of the measurements to surgical procedures, and in turn limit its ability to determine optimal tunnel placement, to assess new protocols for improved tunnel placement and to correlate tunnel positions to biomechanical functions of the knee. They may also limit the usefulness of the data obtainable from retrospective studies. The use of CT scans is preferred in recent studies $^{2}$. However, the postoperative $\mathrm{CT}$ is not available for most ACL reconstructions while intraoperative CT increases the radiation exposure to patients and surgical staff. Intraoperative open MRI and postoperative MRI are time consuming and not a routine procedure for ACL reconstruction nowadays. Thus, they are suitable for retrospective and follow-up patient studies of the long-term effects of tunnel positions on the clinical outcome. Knee scoring systems are frequently used in follow-up studies. They do not include criteria on tunnel position, though graft positioning has been identified as one of the most important factors. To include tunnel position as a parameter in studies of clinical outcomes, it is

Medical Imaging 2012: Image-Guided Procedures, Robotic Interventions, and Modeling,

edited by David R. Holmes III, Kenneth H. Wong, Proc. of SPIE Vol. 8316, 831628

(c) 2012 SPIE · CCC code: 1605-7422/12/\$18 - doi: 10.1117/12.911131

Proc. of SPIE Vol. 8316 831628-1 
necessary to have a tunnel position estimation method that is reliable and repeatable so that clinicians can be assured that the differences in measurements reflect actual variations in surgical placement rather than measurement error ${ }^{3}$.

The goal of the present work is to develop and validate a method to estimate tunnel positions by our single-image contour-based 3D-2D registration using two postoperative X-ray images. To facilitate its use in clinical routine, the two images are not restricted to AP and LAT, as long as they are convenient for tunnels recognition and acquired from the viewpoints not too close to each other. We assume that the intrinsic parameters are known (or estimated by performing a one-time calibration), while the relative imaging position of acquiring the two postoperative X-ray images is unknown.

\section{3D TUNNEL POSITION ESTIMATION}

\subsection{The Tunnel Position Estimation Framework}

A diagram of our framework is shown in Figure 1. The core of the framework is our single-image contour-based 3D$2 \mathrm{D}$ registration algorithm that finds the relative pose of the surface model of knee with respect to the imaging coordinate system, so that we can further estimate the 3D positions of the ACL tunnels with respect to the surface model according to the post-operative X-ray images. The only interactive stage is the tunnel marking, where a surgeon marks the ACL tunnels on the X-ray images. The necessity of this interaction is due to the fact that it is hard to accurately extracted tunnel boundaries in postoperative X-ray images in a pure automatic manner, especially when Hamstring grafts are used.

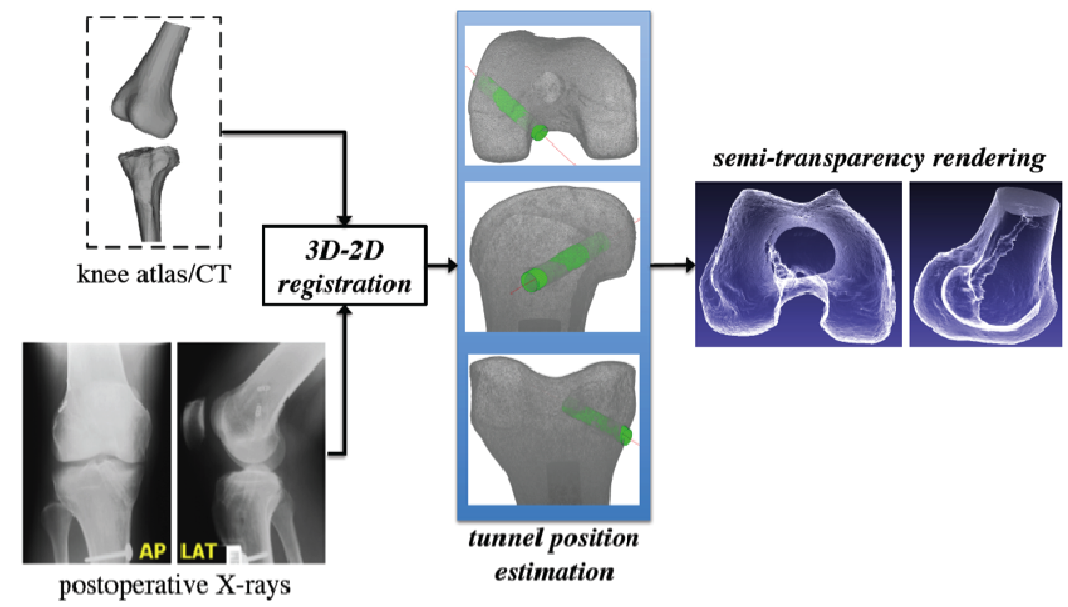

Figure 1. Overview of the 3D tunnel position estimation framework.

The $3 \mathrm{D}$ position of a tunnel is estimated through the following steps. Given a postoperative X-ray image, a surgeon is asked to mark the tunnel boundaries using straight lines. After that, our single-image 3D-2D registration is carried out on that image and one 3D position of the X-ray source is estimated. To estimate the 3D position of a tunnel, the marked tunnel boundaries are first converted into 3D planes (we call them boundary-planes since each of them corresponds to a boundary of the marked tunnel). Then, we compute the plane that bisects the acute dihedral angle of the two boundaryplanes. Performing the same single-image registration and calculation procedure on another X-ray image, we obtain the second bisecting plane. Finally, we calculate the intersection of the two bisecting planes, which is the central axis of the estimated ACL tunnel. As for the entry point of the ACL tunnel, it is calculated as the intersection point of the estimated central axis of the tunnel with the bone surface. Manually outlining the contours of the femur and tibia is timeconsuming and error-prone. On the contrary, automatic contour detectors have good localization performance but generally produce spurs and broken contours. Therefore, a robust and accurate single-image 3D-2D registration method is required especially for its wide deployment in clinical routine. We adopt our previous 3D-2D registration algorithm ${ }^{4,5}$ and extend it to a contour-based registration method for this purpose.

One thing needs to emphasize is that, only single-image 3D-2D registration will be performed using our method on each view, because the X-ray imager is externally uncalibrated and the relative pose of the two images are not available.

\subsection{The Single-Image 3D-2D Registration}

In this section, we extend our previous $3 \mathrm{D}-2 \mathrm{D}$ rigid registration algorithm ${ }^{4,5}$ to a hybrid single-image $3 \mathrm{D}-2 \mathrm{D}$ registration method that uses both the image contour points and the image gradient. In this case, we have a set of $N 2 \mathrm{D}$ 
image points associated with their image gradients, $\left(\mathbf{x}_{n}, \mathbf{g}_{n}\right)$, and $M$ 3D model points associated with their outer normals, $\left(\mathbf{X}_{m}, \mathbf{N}_{m}\right)$. Without knowing the correspondences between them, we are solving the following problem:

$$
\hat{\boldsymbol{\theta}}=\arg \min _{\boldsymbol{\theta}} \sum_{m=1}^{M} \sum_{n=1}^{N} p_{m n}\left\|\left(\mathbf{x}_{n}, \mathbf{g}_{n}\right)-\mathrm{T}\left(\left(\mathbf{X}_{m}, \mathbf{N}_{m}\right) ; \boldsymbol{\theta}\right)\right\|^{2},
$$

where $\mathrm{T}$ is a non-linear mapping that includes a $3 \mathrm{D}$ transformation $\boldsymbol{\theta}=\{\mathbf{R}, \mathbf{t}\}$ and a perceptive projection, and $p_{m n}$ denotes the probability that $\mathbf{x}_{n}$ corresponds to $\mathbf{X}_{m}$. We consider the registration problem in (1) as a probability density estimation problem. The projections of the 3D points represent the centers of a von Mises-Fisher-Gaussian (vMFG) mixture model and the 2D points are observations drawn from the model. The correspondence probability between a $2 \mathrm{D}$ point $\left(\mathbf{x}_{n}, \mathbf{g}_{n}\right)$ and a 3D point $\left(\mathbf{X}_{m}, \mathbf{N}_{m}\right)$ is modeled by a vMFG distribution with mean $\mu_{m}=\mathrm{T}\left(\mathbf{X}_{m} ; \boldsymbol{\theta}\right)$, variance $\sigma^{2}$, central direction $v_{m}=\mathrm{T}\left(\mathbf{N}_{m} ; \boldsymbol{\theta}\right)$ and concentration $\kappa>0$ as

$$
p\left(\mathbf{x}_{n}, \overline{\mathbf{g}}_{n} \mid m\right)=\frac{1}{(2 \pi \sigma)^{2} I_{0}(\kappa)} \exp \left(\kappa \overline{\mathbf{g}}_{n}^{T} \bar{v}_{m}-\frac{\left\|\mathbf{x}_{n}-\mu_{m}\right\|^{2}}{2 \sigma^{2}}\right)
$$

where $\overline{\mathbf{g}}_{n}=\mathbf{g}_{n} /\left\|\mathbf{g}_{n}\right\|, \bar{v}_{m}=v_{m} /\left\|v_{m}\right\|$ and $I_{0}(\kappa)$ denotes the modified Bessel function of the first kind and order 0.

The optimization is solved using an expectation maximization (EM) algorithm by defining the surrogate function

$$
Q\left(\boldsymbol{\theta}, \sigma^{2}, \kappa\right)=\frac{1}{2 \sigma^{2}} \sum_{n=1}^{N} \sum_{M=1}^{M} p_{m n}\left(\left\|\mathbf{x}_{n}-\mathbf{T}\left(\mathbf{X}_{m} ; \boldsymbol{\theta}\right)\right\|^{2}+\log \sigma^{2}\right)-\kappa \sum_{n=1}^{N} \sum_{M=1}^{M}\left(p_{m n} \overline{\mathbf{g}}_{n}^{T} \bar{v}_{m}+\log I_{0}(\kappa)\right) .
$$

Here, the first term is related to the spatial positions, and the second term is about gradient directions. In E-step,

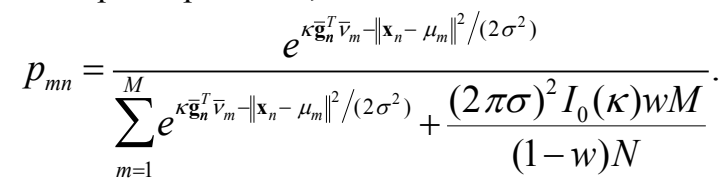

In M-step, the maximum likelihood estimate (MLE) of $\sigma^{2}$ is obtained conditioned on $\boldsymbol{\theta}$ analytically ${ }^{4}$. The expression of $\kappa$ is however given in terms of the ratio of modified Bessel functions, and it is not possible to get an analytical solution. An approximation of $\kappa$ is obtained by iterating the Newton method a few (e.g. 2-3) times through calculating

$$
\kappa^{\text {update }}=\kappa-\frac{A_{2}(\kappa)-\bar{r}}{1-A_{2}^{2}(\kappa)-A_{2}(\kappa) / \kappa}
$$

where $A_{2}(\kappa)=\frac{1}{C} \sum_{m=1}^{M} \sum_{n=1}^{N} p_{m n} \overline{\mathbf{g}}_{n}^{T} \bar{v}_{m}$ and $C=\sum_{m=1}^{M} \sum_{n=1}^{N} p_{m n}$. Therefore, we have the following method:

1) Initialize $\boldsymbol{\theta}^{(0)}$ and $\sigma^{(0)}$, and set $\kappa^{(0)}=0$

2) Compute $p_{m n}^{(t+1)}$

3) Compute $\sigma^{(t+1)}$ and $\boldsymbol{\kappa}^{(t+1)}$ conditioned on $\boldsymbol{\theta}^{(t)}$

4) Compute $\boldsymbol{\theta}^{(t+1)}$ by a numerical optimization approach, conditioned on the updated variance $\sigma^{(t+1)}$ and $\kappa^{(t+1)}$

5) Check convergence.

In our current implementation, Step 4 uses the particle swarm algorithm ${ }^{6}$ to estimate $\boldsymbol{\theta}^{(t+1)}$.

\subsection{The Estimation of 3D Tunnel Positions}

After performing the single-image 3D-2D registration described above respectively on the two X-ray images, 3D tunnel position is estimated using the estimated transformations between the knee model and the X-ray images. Without losing any generality, let us simply start from an arbitrary view of the two, and assume that there is only one tunnel in the image. For multiple tunnels, we merely need to repeat the procedure described in the subsection until all tunnels have been processed. The estimation of a bone tunnel includes the calculations of the tunnel central axis and the tunnel entry point on the inner wall femoral condyle. It is achieved through the following five steps.

Step 1. Two boundaries of a tunnel marked on the image are converted into two 3D planes, which we call "boundary planes" since each one corresponds to one boundary of the tunnel marked in the image. A boundary plane is defined by the estimated X-ray source and two rays that connect the estimated X-ray source with two different points on the 
boundary line in the image (the orange and blue lines in Figure 2). Accordingly, we obtained two boundary planes for one tunnel on that image; the planes in orange and in blue, in Figure 2.

Step 2. We compute the third plane that bisects the acute dihedral angle of these two boundary planes (the plane with black border in the amplified region of Figure 2), and call it "bisecting plane".

Step 3. A second bisecting plane is computed by performing the calculations in Step 1-2 on another X-ray image. Consequently, for one tunnel, we obtained two bisecting planes from two images.

Step 4. We calculate the intersection line (the green solid line in of the two bisecting planes. This intersection line is defined as the "central axis" of the estimated ACL tunnel in 3D space with respect to the femur coordinate system.

Step 5. As for the estimation of the entry point of an ACL tunnel, it is calculated as the 3D intersection of the central axis of the estimated tunnel with the surface of the knee model.

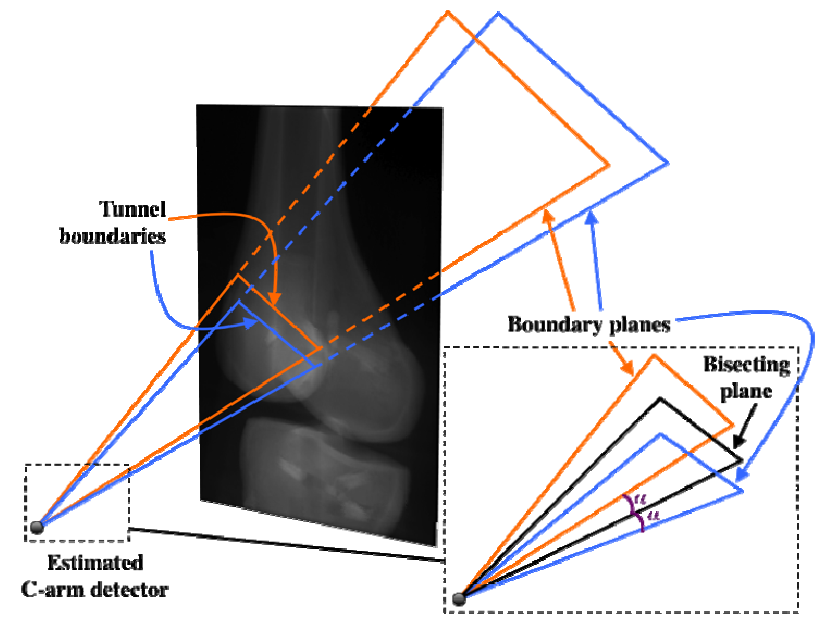

Figure 2. The calculations of boundary planes and their bisecting plane for an image using the estimate of $\mathrm{C}$-arm detector source and the two tunnel boundaries marked on the image.

Ideally, a tunnel can be modeled as a truncated cylinder. In clinical practice, however, a tunnel can be a truncated cone due to the bone tunnel dilation and bone grafting. Our estimation method is applicable without modification for both situations.

\section{EXPERIMENTS AND RESULTS}

To evaluate the performance of the proposed method, a study using digitally reconstructed radiographs (DRRs) of a plastic phantom (see Figure 3) was conducted. A single-bundle ACL reconstruction was performed on a phantom femur by an experienced orthopedic surgeon. CT images of femur and tibia were acquired separately using a custom-made cone-beam CT bench system with voxel size of $0.25 \mathrm{~mm}^{3}$. Two CT data sets were placed in such a position that local coordinate systems of femur and tibia were aligned in parallel and $40 \mathrm{~mm}$ translation in Y direction and merged to generate one CT data containing both femur and tibia by resampling with voxel size of $0.3 \mathrm{~mm}^{3}$. DRR was generated from the merged CT data with known intrinsic and extrinsic parameters that simulate realistic X-ray device setting. The imaging parameters used in this experiment to generate DRR are follows. The image is $1200 \times 600$ pixels with pixel size $0.25 \mathrm{~mm}^{2}$. The detector-source distance was $400 \mathrm{~mm}$ and the detector-object distance was $200 \mathrm{~mm}$. The principal point on the DDR is $(600,800)$ in pixels. The plastic phantom underwent a single bundle ACL reconstruction and an example DRR generated from its CT scan are shown in Figure 3.

To establish the ground truth, the CT images were manually segmented. The ground-truth entry points of the tunnels were extract as follows. As for the femoral tunnel, a cylinder was fitted to its surface model. The major axis of the fitted cylinder is defined as the central axis of the tunnel. The entry point is the intersection point of the central axis with the medial wall of the lateral femoral condyle. That is, the ground truth includes a surface reconstruction of the distal femur, the central axis and the entry point of the femoral tunnel, and a set of DRRs with known X-ray source positions. 
Manually outlining the contours of the distal femur and proximal tibia is time-consuming and error-prone. But clear image contours significantly reduce the challenges for 3D-2D registration algorithm. On the other hand, automatic contour detectors have good localization performance but commonly produce spurs broken contours. In this case, a robust and accurate 3D-2D registration algorithm is required especially for its wide deployment in clinical routine.

In our framework, we employ automatic contour detection and eliminate the cumbersome manual contour extraction. This is because our probabilistic contour-based method is robust to outliers and missing contours, as will be illustrated in our experiment later. Thus, we employed a general-purpose edge detector ${ }^{7}$ to extract image contours automatically.

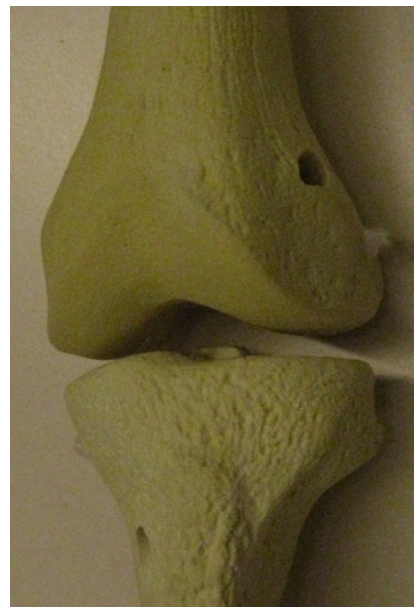

Figure 3. The surface models (middle) and a DRR (
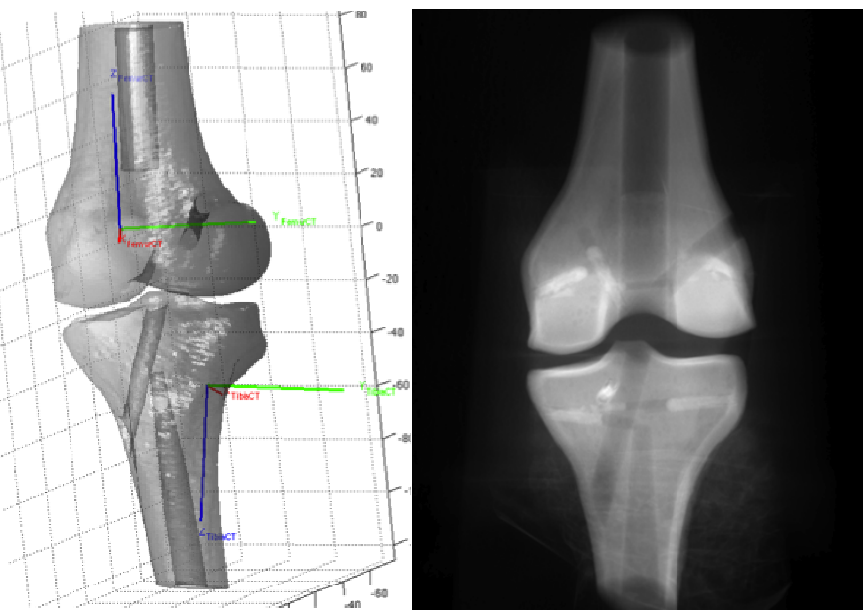

reconstruction (left)

Since the 3D-2D registration is the crucial part of our framework, our experiments focused on the evaluation of its accuracy. The surface model of the femur was used in the experiments, while both the femur and the tibia CT images were used to generate DRRs. The original high resolution CT reconstruction was first downsampled, from 80,300 surface patches to 6,064 surface patches, and then smoothed using Laplacian smoothing. Thus, the surface model used for 3D-2D registration had some differences from the CT reconstruction. The DRRs were generated using the original CT images. These more or less simulated the situation that the actual model used for registration is not as the same as the patient's bone. DRRs generated from 16 different viewpoints within 180 degrees were selected, where the contours were extracted using the SUSAN operator ${ }^{7}$.

For each DRR, 15 trials of single-image 3D-2D registration were carried out using different initializations that were randomly selected from the values that are independently, uniformly distributed within $\pm 10^{\circ}$ and $\pm 10 \mathrm{~mm}$ of the groundtruth. The mean and standard deviation of the registration errors in rotations and translations were listed in Table 1. Using our single-image registration method, the rotations and in-plane translations have been estimated accurately while the off-plane translation (i.e. the depth along Z-direction) had relatively large error. It is known that the depth can be difficult to be accurately estimated for a 3D-2D registration algorithm using one single-image. In addition, by integrating gradient information in a statistical manner, we obtained better accuracy in depth estimation than our previous method ${ }^{5}$.

Table 1. Statistical results using our single-image 3D-2D registration method.

\begin{tabular}{cccccc}
\hline \multicolumn{3}{c}{ Rotation (in degrees) } & \multicolumn{3}{c}{ Translation (in mm) } \\
\hline $\mathrm{X}$ & $\mathrm{Y}$ & $\mathrm{Z}$ & $\mathrm{X}$ & $\mathrm{Y}$ & $\mathrm{Z}$ \\
\hline$-0.43 \pm 1.19$ & $0.45 \pm 2.17$ & $0.23 \pm 1.05$ & $0.03 \pm 0.55$ & $-0.03 \pm 0.54$ & $-2.73 \pm 1.64$ \\
\hline
\end{tabular}

After performing our single-image registrations, two DRRs from approximately AP and LAT views were selected to estimate the tunnel entry point. Following the tunnel position estimation procedure described in Section 2.3, we obtained accurate result with $0.53 \pm 0.30 \mathrm{~mm}$ distance to the ground-truth entry point location. In fact, the estimation of the tunnel entry point location will not be affected too much by the estimation error in depth because, with small rotation and inplane translation errors, the estimation error in depth will influence the size of the dihedral angle of two boundary-planes but have little effect on the bisecting plane. 


\section{CONCLUSION}

Accurate estimation of tunnel position is a prerequisite for evaluating the ACL reconstruction postoperatively, and the confirmation of tunnel placement intraoperatively. The evaluation results, in turn, could be used to design better protocols and evaluation criteria for surgical procedures. The proposed method only requires two X-ray images, which are part of the standard diagnostics procedure in clinical routine, to estimate the tunnel positions. The most significant advantage of the present approach is the elimination of the necessity of acquiring a CT image of the patient. Therefore, our proposed technique is more appropriate for retrospective studies as well as long-term follow-up studies. By providing 3D tunnel location within the knee, it also allows clinicians to analyze the relationship between each tunnel wall or outlet location and the reference landmarks from all viewpoints, including the axial viewpoint.

The preliminary results reported in this paper are very encouraging, and we are planning a more extensive evaluation study. The method promises to be a very useful tool for providing accurate 3D ACL tunnel positioning assessment data intraoperative and postoperatively with minimal X-ray imaging. We also are working on extending the proposed method to a 3D-2D deformable registration method using a statistical shape model. This will greatly facilitate the usefulness of our technique in retrospective studies, where preoperative $\mathrm{CT}$ images are not commonly available.

Acknowledgement: The authors would like to thank Dr. Jeffrey H. Siewerdsen and Dr. Wojciech Zbijewski for their help on acquiring CT images. This research was supported in part by NIH Grant 1-R21-EB007747, in part by Research Grant and RPg Exchange Funding of the University of Hong Kong, and in part by Johns Hopkins University internal funds.

\section{REFERENCES}

[1] B. Forsythe, S. Kopf, A.K. Wong, and et al. The Location of Femoral and Tibial Tunnels in Anatomic DoubleBundle Anterior Cruciate Ligament Reconstruction Analyzed by Three-Dimensional Computed Tomography Models. J Bone Joint Surg Am 92(6), 1418-1426 (2010).

[2] P. Kasten, M. Szczodry, J. Irrgang, and et al. What is the role of intra-operative fluoroscopic measurements to determine tibial tunnel placement in anatomical anterior cruciate ligament reconstruction? Knee Surgery, Sports Traumatology, Arthroscopy 18, 1169-1175 (2010).

[3] T.V.S. Klos, M.K. Harman, R.J.E. Habet, and et al. Locating femoral graft placement from lateral radiographs in anterior cruciate ligament reconstruction: a comparison of 3 methods of measuring radiographic images. Arthroscopy: The Journal of Arthroscopic \& Related Surgery 16(5), 499-504 (2000)

[4] X. Kang, R.H. Taylor, M. Armand, and et al. Correspondenceless 3D-2D Registration Based on Expectation Conditional Maximization, in Proc. SPIE 7964, 79642Z-9642Z-8 (2011).

[5] X. Kang, W.P. Yau, R.H. Taylor, and Y. Otake. A novel method in assessing tunnel positions in ACL reconstruction, in Proc. 11th Annual Meeting of CAOS-International, (2011).

[6] J. Kennedy, R.C. Eberhart, Swarm Intelligence. Morgan Kaufmann. (2001).

[7] S. Smith, J. Brady. SUSAN-A new approach to low-level image processing. Int. J. Computer Vision 23(1), 45-78 (1997). 